\title{
Risk Factors of Delirium in Sequential Sedation Patients in Intensive Care Units
}

\author{
Jie Yang, Yongfang Zhou, Yan Kang, Binbin Xu, Peng Wang, Yinxia Lv, and Zhen Wang \\ Department of Critical Care Medicine, West China Hospital of Sichuan University, Chengdu 610041, China \\ Correspondence should be addressed to Yan Kang; kang_yan_123@163.com
}

Received 1 July 2017; Revised 10 September 2017; Accepted 3 October 2017; Published 31 October 2017

Academic Editor: Paul M. Tulkens

Copyright (C) 2017 Jie Yang et al. This is an open access article distributed under the Creative Commons Attribution License, which permits unrestricted use, distribution, and reproduction in any medium, provided the original work is properly cited.

Background. Delirium is a primary adverse event in ventilated patients who receive long-term monosedative treatment. Sequential sedation may reduce these adverse effects. This study evaluated risk factors for delirium in sequential sedation patients. Methods. A total of 141 patients who underwent sequential sedation were enrolled. Delirium was diagnosed using Confusion Assessment Method for the Intensive Care Unit (CAM-ICU) scale. Univariate and multivariate Cox proportional hazards regressions were used to predict risk factors. Results. Older age $(\geq 51)(\mathrm{RR}=2.432,95 \% \mathrm{CL} 1.316-4.494, p=0.005)$, higher SOFA score $(\geq 14)(\mathrm{RR}=$ 2.022, 95\% CL 1.076-3.798, $p=0.029)$, regular smoking ( $\mathrm{RR}=2.366,95 \%$ CL 1.277-4.382, $p=0.006$ ), and higher maintenance dose of midazolam $(\mathrm{RR}=1.052,95 \% \mathrm{CL} 1.000-1.107, p=0.049)$ and fentanyl $(\mathrm{RR}=1.045,95 \%$ CL $1.019-1.072, p=0.001)$ when patients met sequential criteria, were independent risk factors of delirium. Sequential sedation with dexmedetomidine $(R R=0.448$, 95\% CL 0.209-0.963, $p=0.040$ ) was associated with a lower risk of delirium. Conclusions. Older age, higher SOFA score, regular smoking, and higher maintenance dose of midazolam and fentanyl when patients met sequential criteria were independent risk factors of delirium in sequential sedation patients. Sequential sedation with dexmedetomidine reduced risk of delirium.

\section{Introduction}

Delirium is a disorder of patient consciousness that is characterized by four aspects: an acute change in patient mental status, loss of attention, disturbance in thinking, and cognitive dysfunction [1]. Delirium results from various causes in intensive care unit (ICU) patients.

Previous studies demonstrated that patients under mechanical ventilation exhibit a higher risk of experiencing delirium than nonmechanically ventilated patients $(20 \%-50 \%)$ [2]. The occurrence of delirium is also more frequent in elderly patients [3-5], and physicians often have difficulty identifying delirium, which leads to the unreasonable management of ICU patients [6]. Therefore, delirium generally results in poor outcomes in ICU patients, including prolonged duration of mechanical ventilation, increased length of stay, higher mortality, and greater cost [7-9].

The administration of analgesic sedatives to long-term mechanically ventilated patients is an effective means of relieving anxiety and agitation [10]. Midazolam and propofol are generally used for sedation [11-13]. However, these sedatives exhibit some adverse effects when used alone for long-term sedation, such as withdrawal reactions, delayed extubation from drug accumulation, hypertriglyceridemia, respiratory depression, and circulative function depression $[14,15]$. Delirium is probably associated with these adverse effects, and it is also a main effect in ICU patients [13]. Sequential sedative use may reduce these adverse effects and lower the risk of delirium [16].

The risk factors of delirium in ICU patients are different among studies but generally include older age, higher acute physiology and chronic health evaluation (APACHE) II score, and exposure to sedatives and analgesics. However, few current studies examined delirium in patients who received sequential sedation. Therefore, the present study investigated the factors of delirium in patients who received sequential sedation in the ICU.

\section{Materials and Methods}

2.1. Patients. In this planned study, we selected patients from a previous study (ClinicalTrials.gov Identifier NCT02528513) 


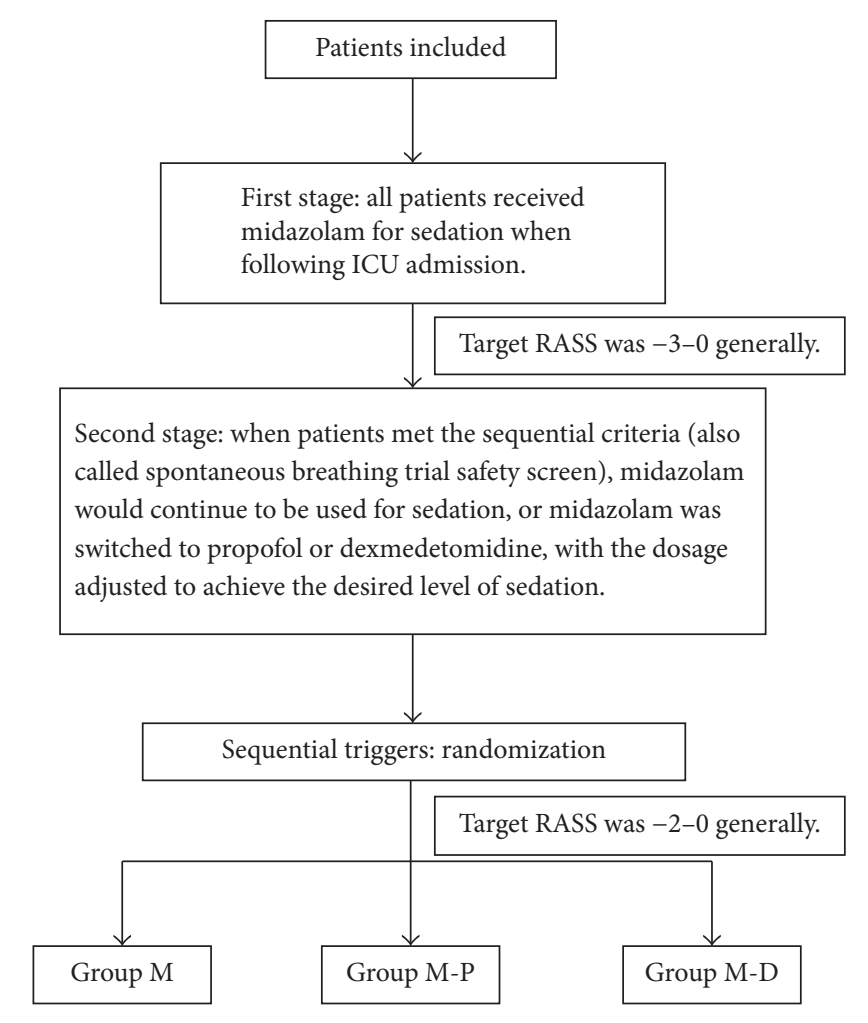

FIGURE 1: Sequential sedation process.

during December 2015 to January 2017 in medical and surgical ICU in the West China Hospital of Sichuan University, Sichuan, China. The ethics committee of West China Hospital of Sichuan University approved this study. All of the patients involved in the trial signed an informed consent form and consented to participate in the study as appropriate. All patients older than 18 years old and younger than 80 years old, who were expected to receive mechanical ventilation longer than 72 hours and accepted sedation therapy, were recruited on admission to the ICU. Patients were excluded if they had a history of sedatives allergy (propofol, midazolam, or dexmedetomidine) and significant neurological diseases that would confound the evaluation of delirium, chronic renal failure, severe organ dysfunction, history of alcoholism, and taking antianxiety drugs or hypnotics.

All patients in this study received continuous intravenous fentanyl for analgesia following ICU admission. Patient sedation treatment was divided into three groups (Figure 1): (1) the midazolam group (group M) throughout the sedative period until extubation; (2) the sequential use of midazolam and propofol group (group M-P), in which midazolam was switched to propofol when patients met sequential criteria; and (3) the sequential use of midazolam and dexmedetomidine group (group M-D), in which midazolam was switched to dexmedetomidine when patients met sequential criteria. The Richmond agitation-sedation scale (RASS) was used to assess the sedative level in each group [17]. Nurses continuously monitored sedation depth and adjusted the doses of sedative and analgesic drugs according to local sedation procedures to maintain the sedation level to an appropriate degree until patients successfully passed the spontaneous breathing trial (SBT) and were extubated $[13,18]$.

Sequential criteria are also called a spontaneous breathing trial safety screen. A patient's condition was severe following ICU admission. Patients were tested using the spontaneous breathing trial safety screen when their condition improved after a period of treatment. Enrolled patients passed the spontaneous breathing trial (SBT) safety screen if they exhibited adequate oxygenation (oxygen partial pressure $\geq 60 \mathrm{mmHg}$ on a fraction of inspired oxygen $\leq 50 \%$ and a positive endexpiratory pressure $\leq 8 \mathrm{cmH}_{2} \mathrm{O}$ ), stable hemodynamics with no evidence of myocardial ischemia, and no significant use of vasopressors (dopamine or dobutamine $\leq 5 \mu \mathrm{g} / \mathrm{kg} / \mathrm{min}$ or norepinephrine $\leq 2 \mu \mathrm{g} / \mathrm{min}$ ) [19]. However, patients failed the SBT trial if they underwent a 30-minute SBT with $8 \mathrm{cmH}_{2} \mathrm{O}$ pressure support ventilation, $5 \mathrm{cmH}_{2} \mathrm{O}$ positive end-expiratory pressure, and $40 \%$ fraction of inspired oxygen [20].

2.2. Clinical Data. Patient demographic characteristics included age, gender, body mass index (BMI), allergic history, drinking and smoking status, and medical history, including the presence or absence of hypertension. APACHE II score, sequential organ failure assessment score (SOFA), and laboratory test results were determined within 24 hours of ICU admission. Other important information included the use of sedative and analgesic medications, mechanical ventilation status, and sequential sedation characteristics.

2.3. Delirium Assessment. The primary endpoint of this study was the occurrence of delirium. Patient evaluations were implemented using a CAM-ICU scale every 4 hours daily for a maximum of 28 days or until ICU discharge, whichever occurred first [8]. The CAM-ICU includes four parts: abrupt change in mental status, lack of attention, thinking disorder and change in consciousness level [1]. The research team performed delirium assessments daily and recorded the data synchronously.

2.4. Statistical Analysis. Statistical analyses were performed using SPSS 23.0 (Statistical Product and Service Solutions, IBM, USA). Continuous variables are presented as medians and interquartile ranges according to their distribution. Categorical variables are presented as percentages. The Mann-Whitney $U$ test was used to compare differences in continuous variables between delirium and no delirium groups. Chi-square and Fisher's exact probability tests were used to compare differences in categorical variables between two groups. Risk factors were analyzed using Cox proportional hazards regression. Any variables that exhibited $p<$ 0.2 after univariate Cox proportional regression analysis or potential variables associated with delirium were included in the multivariate Cox proportional regression analysis. The cut-off point of age, APACHE II score, and SOFA score were determined using interquartiles to achieve the best discrimination between groups with or without delirium. $p<$ 0.05 was considered statistically significant.

The sample size for this study was estimated according to the incidence of delirium from previous studies that revealed 


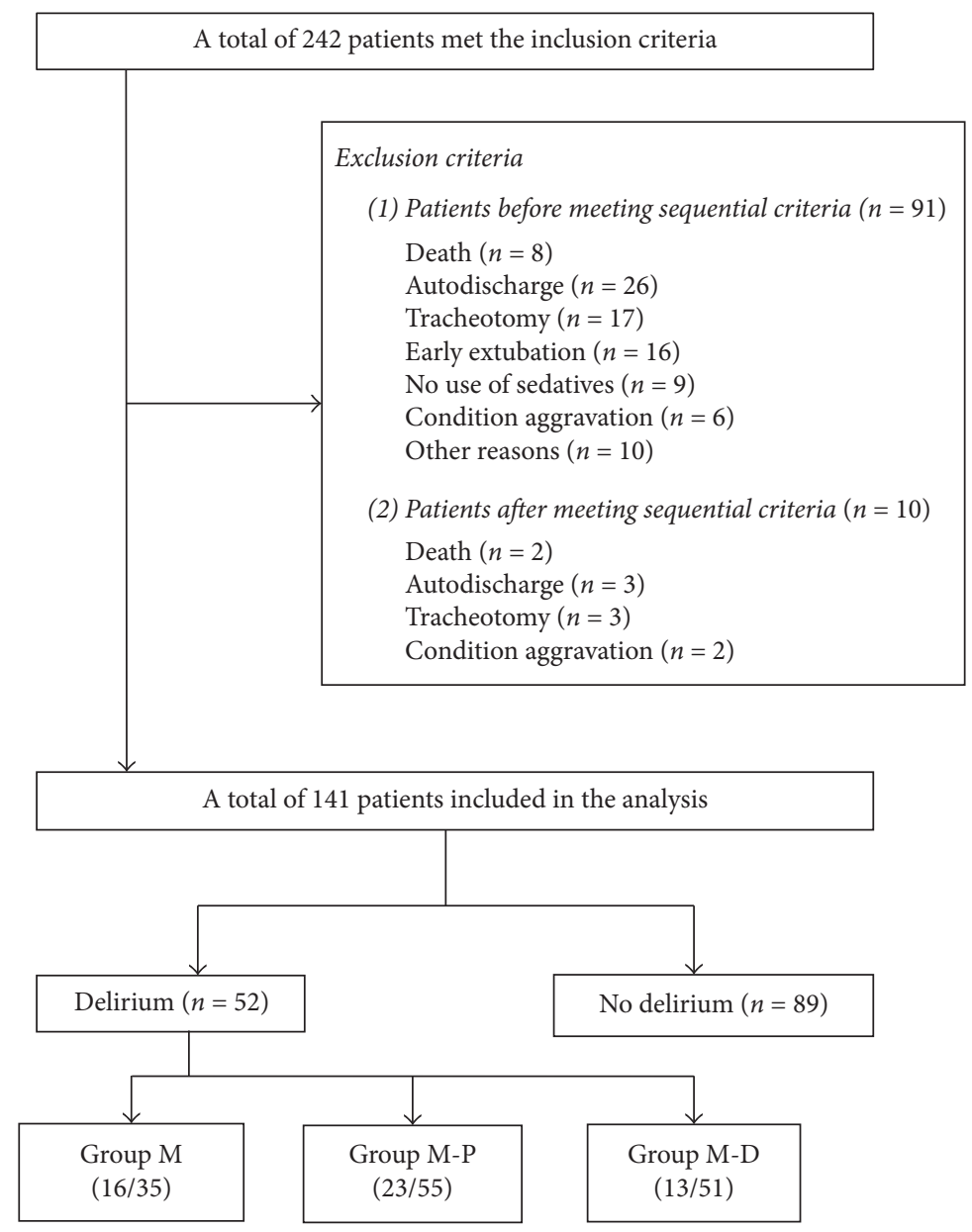

Figure 2: Study flow chart.

a historical ICU delirium incidence between 30\% and 53.8\% $[21,22]$. The sample size limited the number of variables in the multivariate regression model, following the generally accepted rule of one variable per ten patients [23]. We estimated that the final multivariate regression model would include approximately 5 variables. Therefore, we estimated a delirium group minimum sample size of 50 patients. We finally estimated that at least 100 patients should be included in this study based on historical delirium incidence and the delirium group minimum sample size.

\section{Results}

A total of 242 met our inclusion criteria. Ninety-one patients were excluded before meeting the sequential criteria, including 8 patients for death, 26 patients for autodischarge, 17 patients for tracheotomy, 16 patients for early extubation, 9 patients for no use of sedatives, 6 patients for condition aggravation, and another 10 patients for other reasons. The remaining 10 patients met the sequential criteria and received assigned sedatives, but these patients were excluded for death, tracheotomy, autodischarge, and condition aggravation. A total of 141 patients were recruited for the analysis: 35 patients in group M, 55 patients in group M-P, and 51 patients in group
M-D. Figure 2 presents the study flow. There were 52 patients diagnosed with delirium in total. Sixteen patients developed delirium in group $M(16 / 35)$, and 23 patients developed delirium in group M-P (23/55), and 13 patients developed delirium in group M-D (13/51).

Table 1 presents demographic data and baseline characteristics of patients with or without delirium. Delirious patients were significantly older (54 (47-65) versus 49 (38-61), $p=0.023$ ), exhibited higher SOFA scores at ICU admission $(\geq 14)$ (34.6\% versus 19.1\%, $p=0.040)$, and have higher maintenance dose of midazolam $(\mathrm{mg} / \mathrm{kg} / \mathrm{d})(1.896$ (1.440-2.424) versus $1.440(0.960-1.992), p=0.001)$ and a higher maintenance dose of fentanyl when patients met sequential criteria $(\mu \mathrm{g} / \mathrm{kg} / \mathrm{d})(19.32(14.40-24.24)$ versus 18.24 (14.40-21.12), $p=0.035)$.

Univariate Cox proportional regression (Table 2) revealed that age $\geq 51$, regular smoking, SOFA score $\geq 14$, the first dose of fentanyl at ICU admission, and maintenance dose of midazolam and fentanyl increased the risk of developing delirium, whereas sequential sedation using different sedatives may reduce delirium risk. Multivariate Cox proportional regression analysis (Table 3 ) revealed that older age $(\geq 51)(\mathrm{RR}=2.432,95 \% \mathrm{CL} 1.316-4.494, p=0.005)$, higher SOFA score $(\geq 14)(\mathrm{RR}=2.022,95 \%$ CL 1.076-3.798, 
TABLE 1: Characteristics of patients with and without delirium in this study.

\begin{tabular}{|c|c|c|c|}
\hline Variables & Delirium $(n=52)$ & Nondelirium $(n=89)$ & $p$ \\
\hline \multicolumn{4}{|l|}{ Baseline characteristics of patients } \\
\hline Age (years) & $54(47-65)$ & $49(38-61)$ & $0.023^{\text {** }}$ \\
\hline$<51$ & $19(36.5)$ & $51(57.3)$ & $0.017^{\text {** }}$ \\
\hline$\geq 51$ & $33(63.5)$ & $38(42.7)$ & \\
\hline Male $(n, \%)$ & $38(73.1)$ & $56(62.9)$ & 0.217 \\
\hline Body mass index (BMI) & $23.35(20.24-26.70)$ & $23.77(21.86-25.79)$ & 0.632 \\
\hline Allergic history $(n, \%)$ & & & 0.358 \\
\hline Yes & $3(5.8)$ & $2(2.2)$ & \\
\hline No & $49(94.2)$ & $87(97.8)$ & \\
\hline Regular drinking $(n, \%)$ & & & 0.838 \\
\hline Yes & $19(36.5)$ & $31(34.8)$ & \\
\hline No & $33(63.5)$ & $58(65.2)$ & \\
\hline Regular smoking $(n, \%)$ & & & 0.175 \\
\hline Yes & $21(40.4)$ & $26(29.2)$ & \\
\hline No & $31(59.6)$ & $63(73.8)$ & \\
\hline Hypertension $(n, \%)$ & & & 0.525 \\
\hline Yes & $11(21.2)$ & $15(16.9)$ & \\
\hline No & $41(78.8)$ & $74(83.1)$ & \\
\hline ICU admission diagnosis $(n, \%)$ & & & 0.240 \\
\hline Pneumonia & $13(25.0)$ & $22(24.7)$ & \\
\hline Sepsis & $11(21.2)$ & $20(22.5)$ & \\
\hline Trauma & $15(28.8)$ & $15(16.9)$ & \\
\hline Pancreatitis & $13(25.0)$ & $27(30.3)$ & \\
\hline other digestive disease & $0(0.0)$ & $5(5.6)$ & \\
\hline \multicolumn{4}{|l|}{ (1) Patients at ICU admission (before meeting the sequential criteria) } \\
\hline APACHE II & $19(14-25)$ & $19(14-23)$ & 0.771 \\
\hline$<23$ & $38(73.1)$ & $68(76.4)$ & 0.659 \\
\hline$\geq 23$ & $14(26.9)$ & $21(23.6)$ & \\
\hline SOFA score & $10(8-14)$ & $10(7-13)$ & 0.595 \\
\hline$<14$ & $34(65.4)$ & $72(80.9)$ & $0.040^{\text {** }}$ \\
\hline$\geq 14$ & $18(34.6)$ & $17(19.1)$ & \\
\hline Cholesterol (mmol/L) & $2.31(1.86-3.28)$ & $2.29(1.44-3.07)$ & 0.082 \\
\hline Triglyceride (mmol/L) & $1.41(0.79-2.43)$ & $1.40(0.82-2.21)$ & 0.584 \\
\hline The first dose of midazolam $(\mathrm{mg} / \mathrm{kg} / \mathrm{d})$ & $1.728(1.380-2.184)$ & $1.680(1.320-1.920)$ & 0.145 \\
\hline The first dose of fentanyl $(\mu \mathrm{g} / \mathrm{kg} / \mathrm{d})$ & $18.48(15.36-23.04)$ & $17.28(14.40-19.92)$ & 0.068 \\
\hline \multicolumn{4}{|l|}{ (2) After meeting the sequential criteria } \\
\hline The maintenance dose of midazolam (mg/kg/d) & $1.896(1.440-2.424)$ & $1.440(0.960-1.992)$ & $0.001^{* *}$ \\
\hline The maintenance dose of fentanyl $(\mu \mathrm{g} / \mathrm{kg} / \mathrm{d})$ & $19.32(14.40-24.24)$ & $18.24(14.40-21.12)$ & $0.035^{\text {×* }}$ \\
\hline The accumulated dose of midazolam (mg) & $336(200-601)$ & $400(200-750)$ & 0.603 \\
\hline The accumulated dose of fentanyl (mg) & $4.315(2.5-7.5)$ & $4.250(3.0-7.0)$ & 0.710 \\
\hline Blood glucose $(\mathrm{mmol} / \mathrm{L})$ & $7.515(5.930-11.035)$ & $8.575(6.870-10.215)$ & 0.257 \\
\hline Triglyceride (mmol/L) & $1.445(1.020-2.400)$ & $1.860(1.245-2.415)$ & 0.201 \\
\hline \multicolumn{4}{|c|}{ (3) Continuous use of midazolam alone or sequential use of midazolam and propofol/dexmedetomidine after meeting the sequential criteria } \\
\hline Time of meeting the sequential criteria to stop sedation (hours) & $33.00(20.75-56.75)$ & $25.00(19.75-47.25)$ & 0.243 \\
\hline Time of meeting the sequential criteria for extubation (hours) & $48.75(24.85-70.0)$ & $34.75(24.10-50.25)$ & 0.317 \\
\hline Time of MV (hours) & $144.75(109.00-200.25)$ & $160.00(109.50-209.50)$ & 0.346 \\
\hline ICU length of stay (days) & $12.91(9.89-18.89)$ & $14.85(9.90-19.98)$ & 0.462 \\
\hline Hospital length of stay (days) & $22.33(15.32-40.06)$ & $19.92(14.85-30.52)$ & 0.357 \\
\hline NIPPV after extubation $(n, \%)$ & & & 0.481 \\
\hline Yes & $14(26.9)$ & $29(32.6)$ & \\
\hline No & $38(73.1)$ & $60(67.4)$ & \\
\hline
\end{tabular}


TABLe 1: Continued.

\begin{tabular}{lcc}
\hline Variables & Delirium $(n=52)$ & Nondelirium $(n=89)$ \\
\hline Vasoactive agent $(n, \%)$ & & $29(32.6)$ \\
Yes & $13(26.0)$ & $60(67.4)$ \\
No & $37(74.0)$ & \\
Sequential sedatives $(n, \%)$ & & $19(21.3)$ \\
Midazolam (group M-M) & $16(30.8)$ & $32(36.0)$ \\
Propofol (group M-P) & $23(44.2)$ & $38(42.7)$ \\
Dexmedetomidine (group M-D) & $13(25.0)$ & 0.101 \\
\hline
\end{tabular}

BMI, body mass index; APACHE, acute physiology and chronic health evaluation; SOFA, sequential organ failure assessment; MV, mechanical ventilation; NIPPV, noninvasive positive pressure ventilation; ${ }^{\text {** }} p<0.05$.

TABLE 2: Univariate Cox proportional regression of ICU delirium in sequential sedation patients.

\begin{tabular}{|c|c|c|c|}
\hline Variables & $\mathrm{RR}$ & 95\% CL & $p$ \\
\hline Age $(\geq 51)$ & 1.957 & $1.112-3.444$ & $0.020^{\text {** }}$ \\
\hline Allergic history & 2.013 & $0.626-6.467$ & 0.240 \\
\hline Regular drinking & 1.078 & $0.613-1.895$ & 0.795 \\
\hline Regular smoking & 1.580 & $0.908-2.750$ & $0.106^{*}$ \\
\hline Hypertension & 1.249 & $0.642-2.430$ & 0.513 \\
\hline APACHE II $(\geq 23)^{\mathrm{a}}$ & 1.146 & $0.621-2.115$ & 0.663 \\
\hline SOFA score $(\geq 14)^{\mathrm{a}}$ & 1.801 & $1.016-3.190$ & $0.044^{\text {** }}$ \\
\hline The first dose of midazolam $(\mathrm{mg} / \mathrm{kg} / \mathrm{d})^{\mathrm{a}}$ & 1.095 & $0.833-1.438$ & 0.515 \\
\hline The first dose of fentanyl $(\mu \mathrm{g} / \mathrm{kg} / \mathrm{d})^{\mathrm{a}}$ & 1.040 & $1.004-1.077$ & $0.030^{\text {** }}$ \\
\hline The maintenance dose of midazolam $(\mathrm{mg} / \mathrm{kg} / \mathrm{d})^{\mathrm{b}}$ & 1.048 & $1.001-1.097$ & $0.043^{\text {x* }}$ \\
\hline The maintenance dose of fentanyl $(\mu \mathrm{g} / \mathrm{kg} / \mathrm{d})^{\mathrm{b}}$ & 1.025 & $1.004-1.047$ & $0.021^{\text {** }}$ \\
\hline The accumulated dose of midazolam (mg) ${ }^{\mathrm{b}}$ & 1.000 & $0.999-1.001$ & 0.960 \\
\hline The accumulated dose of fentanyl (mg) ${ }^{\mathrm{b}}$ & 0.968 & $0.916-1.023$ & 0.252 \\
\hline Time of patients meeting sequential criteria to stop sedation ${ }^{c}$ & 1.003 & $0.995-1.011$ & 0.486 \\
\hline Time of patients meeting sequential criteria for extubation ${ }^{c}$ & 1.002 & $0.995-1.009$ & 0.635 \\
\hline \multicolumn{4}{|l|}{ Sequential sedatives ${ }^{c}$} \\
\hline Midazolam (group M-M) & 1.000 & Ref. & \\
\hline Propofol (group M-P) & 0.863 & $0.456-1.634$ & 0.652 \\
\hline Dexmedetomidine (group M-D) & 0.451 & $0.217-0.939$ & $0.033^{\text {** }}$ \\
\hline
\end{tabular}

${ }^{a}$ Patients at ICU admission (before meeting the sequential criteria). ${ }^{b}$ When patients met the sequential criteria. ${ }^{c}$ After patients met the sequential criteria for extubation. ${ }^{*} p<0.2,{ }^{* *} p<0.05$.

TABLE 3: Multivariate Cox proportional regression of ICU delirium in sequential sedation patients.

\begin{tabular}{|c|c|c|c|}
\hline Risk factors & $\mathrm{RR}$ & $95 \% \mathrm{CL}$ & $p$ \\
\hline Age $(\geq 51)$ & 2.432 & $1.316-4.494$ & $0.005^{\text {** }}$ \\
\hline Regular smoking & 2.366 & $1.277-4.382$ & $0.006^{\text {** }}$ \\
\hline SOFA score $(\geq 14)^{\mathrm{a}}$ & 2.022 & $1.076-3.798$ & $0.029^{\times *}$ \\
\hline The first dose of fentanyl $(\mu \mathrm{g} / \mathrm{kg} / \mathrm{d})^{\mathrm{a}}$ & 0.992 & $0.951-1.035$ & 0.772 \\
\hline The maintenance dose of midazolam $(\mathrm{mg} / \mathrm{kg} / \mathrm{d})^{\mathrm{b}}$ & 1.052 & $1.000-1.107$ & $0.049^{\text {** }}$ \\
\hline The maintenance dose of fentanyl $(\mu \mathrm{g} / \mathrm{kg} / \mathrm{d})^{\mathrm{b}}$ & 1.045 & $1.019-1.072$ & $0.001^{\text {** }}$ \\
\hline \multicolumn{4}{|l|}{ Sequential sedatives ${ }^{c}$} \\
\hline Midazolam (group M-M) & 1.000 & Ref. & \\
\hline Propofol (group M-P) & 0.706 & $0.364-1.369$ & 0.303 \\
\hline Dexmedetomidine (group M-D) & 0.448 & $0.209-0.963$ & $0.040^{\text {×* }}$ \\
\hline
\end{tabular}

${ }^{a}$ Patients at ICU admission (before meeting the sequential criteria). ${ }^{b}$ When patients met the sequential criteria. ${ }^{c}$ After patients met the sequential criteria for extubation. ${ }^{* *} p<0.05$. 
$p=0.029)$, regular smoking $(\mathrm{RR}=2.366,95 \% \mathrm{CL}$ 1.277-4.382, $p=0.006$ ), higher maintenance dose of midazolam $(\mathrm{RR}=1.052,95 \% \mathrm{CL} 1.000-1.107, p=0.049)$, and higher maintenance dose of fentanyl $(\mathrm{RR}=1.045,95 \% \mathrm{CL}$ 1.019-1.072, $p=0.001$ ) were independent risk factors of developing delirium in patients with sequential sedation. Sequential sedation with dexmedetomidine ( $R R=0.448,95 \%$ CL $0.209-0.963, p=0.040$ ) was associated with lower risk of delirium compared to midazolam.

\section{Discussion}

ICU patients exhibit a high incidence of the occurrence of delirium [22, 24, 25]. The range of reported delirium incidences is wide, which is likely due to the use of different patients, objectives, and designs in studies. The total incidence of delirium was $37 \%$ in the present study. This incidence is generally consistent with the incidences reported in previous studies [22, 24, 26].

Many risk factors of delirium were identified in numerous relevant studies, such as older age, hypertension, respiratory disorder, alcohol abuse, smoking, greater illness severity, dementia, and medications [6, 27]. Our study bolstered these opinions about the risk factors of delirium. However, our study was the first to demonstrate how sequential sedation management affected the development of delirium and to identify the risk factors associated with delirium.

Age is the most basic characteristic for patients, but older age is a predisposing risk factor for ICU delirium [28-30]. The present study found that patients older than 51 were at almost 2.4 times $(\mathrm{RR}=2.432, p=0.005)$ the risk for development of delirium than patients younger than 51. This difference means that delirium developed easily in older ICU patients who received sedation therapy. Some patient's living habits cannot also be ignored, such as alcohol abuse and regular smoking. Alcohol abuse and smoking are well-known risk factors for ICU delirium $[28,31]$. Our study did not find that history of alcohol abuse affected the development of delirium. However, patients with regular smoking had a higher risk for developing delirium $(R R=2.366, p=0.006)$.

Previous studies demonstrated that the development of delirium was associated with features of the acute illness, such as categories of acute illness and the disease severity of individual patients [21,31,32]. APACHE II and SOFA scores are two valid tools to evaluate the disease severity in the ICU $[33,34]$. We did not find an association between delirium and APACHE II score in the final multivariate regression model. However, admission SOFA score was a significantly independent predictor for the development of delirium in multivariate regression analysis. We found that the prevalence of a SOFA score $\geq 14$ was significantly higher in patients with delirium than patients without delirium (34.6\% versus $19.1 \%, p=0.040$ ).

Sedation and analgesia management is often required in ICU patients to relieve anxiety, pain, or other physical discomfort [15]. However, benzodiazepine use is generally related to prolonged durations of MV and the length of ICU stay [35]. Benzodiazepines are also associated with sleep disturbance, posttraumatic stress disorder, and depression

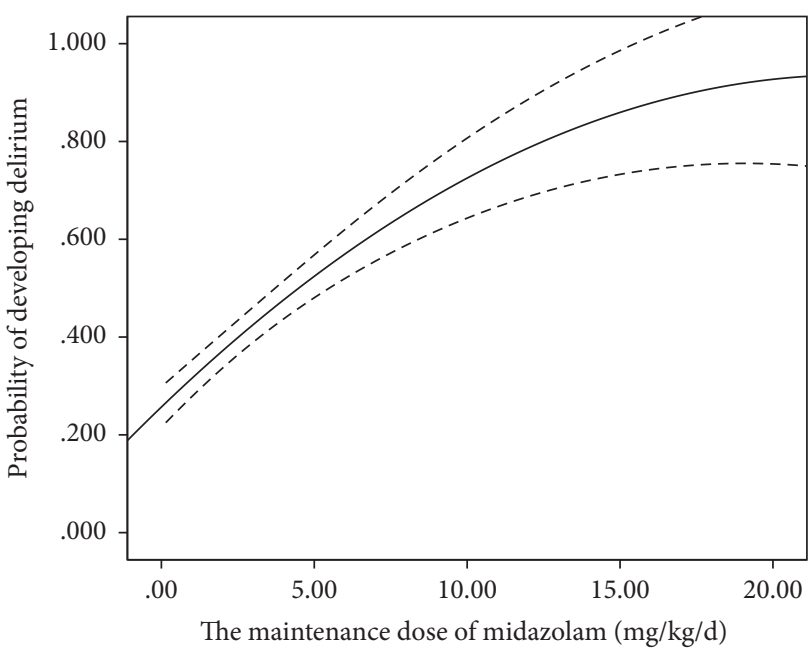

FIGURE 3: The maintenance dose of midazolam when patients met the sequential criteria and the probability of developing delirium. The probability of delirium increased with the maintenance dose of midazolam.

in ICU patients [36, 37]. These factors may lead to the development of delirium in ICU patients [28]. Higher doses of benzodiazepines and fentanyl increased the risk of delirium in burn and trauma ICU patients [38, 39]. Researchers found that benzodiazepine use in the cardiovascular ICU also increased the risk of delirium [24]. Our results demonstrated that benzodiazepine and fentanyl use was reliably related to the development of delirium. We further found that the maintenance doses of midazolam and fentanyl were associated with the development of delirium when patients met the sequential criteria. Patients were at increased risk of delirium when exposed to higher midazolam maintenance $(\mathrm{RR}=1.052, p=0.049)$ (Figure 3). Similarly, higher fentanyl maintenance also increased the risk of developing delirium $(\mathrm{RR}=1.045, p=0.001)$ (Figure 4). Therefore, maintenance doses of midazolam and fentanyl may be reduced appropriately before patients satisfy the sequential criteria and sequentially receive another sedative when their condition has improved. However, benzodiazepines may be used in larger doses for alcohol withdrawal if patients had a serious history of alcohol abuse [6].

Nonpharmacological therapies and pharmacological management are used to prevent the development of delirium to protect against the adverse effects of delirium. Alpha-2 agonists (dexmedetomidine) decrease the incidence of delirium [40-42]. This study used dexmedetomidine for sequential sedation, except when midazolam and propofol were used. Multivariate regression analysis found that sequential sedation with dexmedetomidine was an actually protective factor against delirium $(\mathrm{RR}=0.448, p=0.040)$, and the percentage of developing delirium was lowest in group M-D (Figure 5). This result demonstrates that patients benefit from the sequential use of midazolam and dexmedetomidine, and it reduces the risk for developing delirium. The pharmacology of dexmedetomidine is different from that of benzodiazepines and propofol. It is an 


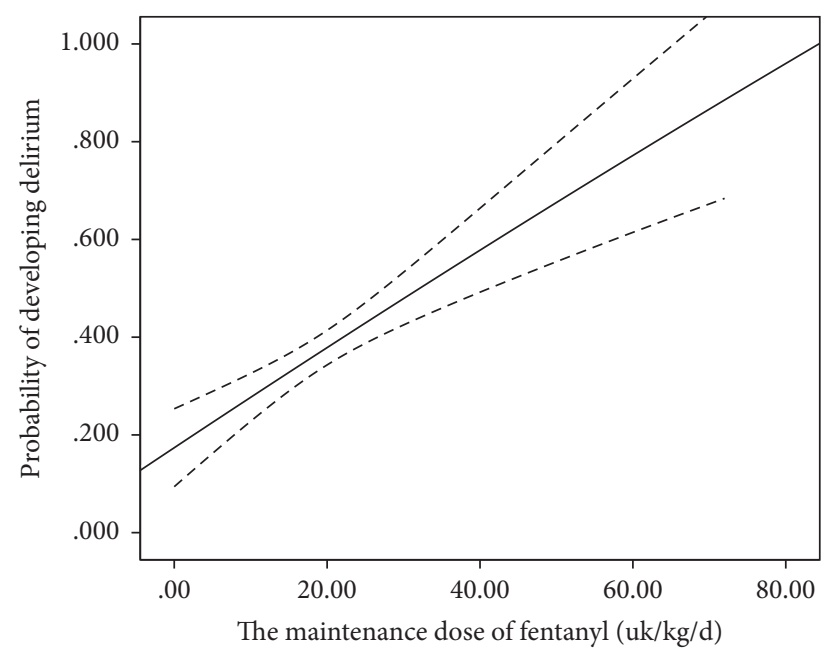

FIGURE 4: The maintenance dose of fentanyl when patients met the sequential criteria and the probability of developing delirium. The probability of delirium also increased with the maintenance dose of fentanyl.

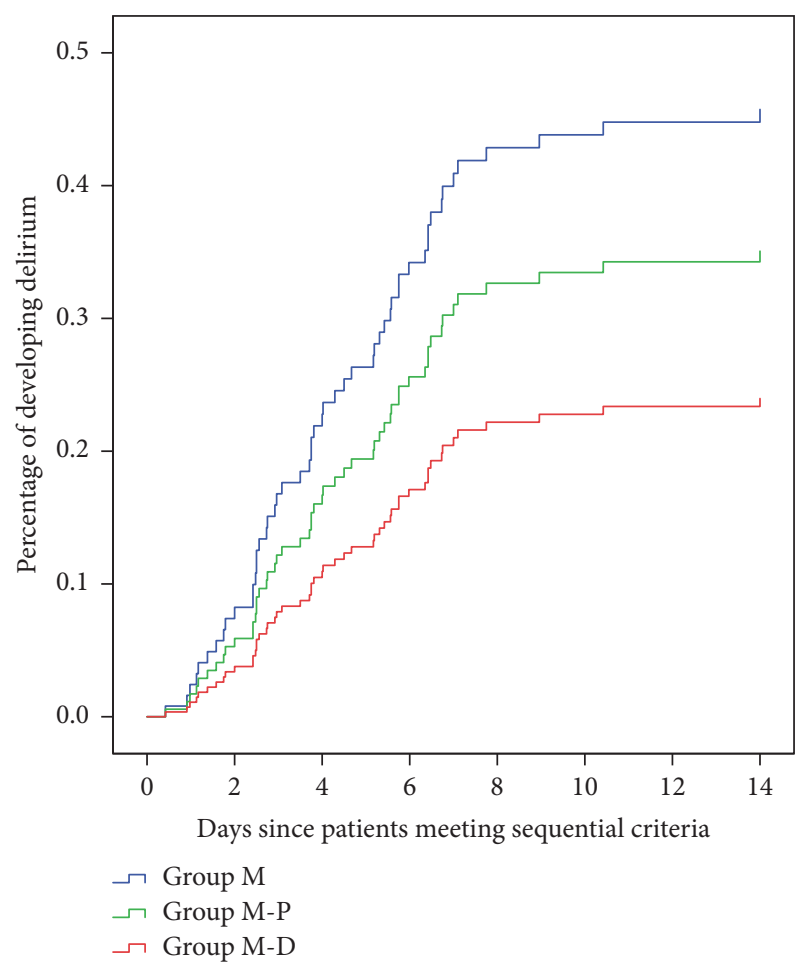

FIGURE 5: The percentage of developing delirium for sequential sedation patients in group $\mathrm{M}$, group $\mathrm{M}-\mathrm{P}$, and group $\mathrm{M}-\mathrm{D}$ (adjusted other variables in multivariate Cox proportional regression).

$\alpha 2$-receptor agonist that provides antianxiety and analgesia by acting on the nucleus coeruleus [43]. Patients who receive dexmedetomidine generally exhibit light sedation and are more cooperative, communicative, and arousable [44]. Sedation with dexmedetomidine improves sleep quality and decreases the incidence of complications [42]. The abovementioned characteristics are likely associated with delirium prevention. The use of benzodiazepines and propofol alone for long-term sedation is associated with some adverse effects $[13,16]$, and these effects may lead to the development of delirium. Sequential use may reduce these adverse effects. This study examined the characteristics of sequential sedation and pharmacology of dexmedetomidine and found that the sequential use of midazolam and dexmedetomidine for long-term sedation was an effective and safe strategy to prevent delirium in ICU patients.

To the best of our knowledge, this report is the first study to evaluate the potential risk factors for the development of delirium in patients who receive sequential sedation. However, our study has some limitations, including small sample size and potential biases inherent to research. More similar studies are needed to confirm our results.

\section{Conclusions}

The present study demonstrated that older age $(\geq 51)$, regular smoking, higher SOFA score $(\geq 14)$, and increased maintenance of midazolam and fentanyl when patients met sequential criteria were significant risk factors of delirium in patients who received sequential sedation. The results of this study also demonstrated that sequential sedation with dexmedetomidine was a protective method to prevent delirium. Our results suggest that management strategies may sometimes require alteration to reduce the incidence and severity of delirium in sequential sedation patients.

\section{List of Abbreviations}

MV: $\quad$ Mechanical ventilation

ICU: Intensive care unit

CAM-ICU: Confusion Assessment Method for the Intensive Care Unit

SBT: $\quad$ Spontaneous breathing trial

BMI: $\quad$ Body mass index

APACHE: Acute physiology and chronic health evaluation

RASS: $\quad$ Richmond agitation-sedation scale

SOFA: Sequential organ failure assessment

NIPPV: Noninvasive positive pressure ventilation.

\section{Ethical Approval}

The ethics committee of West China Hospital of Sichuan University approved the study.

\section{Conflicts of Interest}

The authors declare that they have no conflicts of interest.

\section{Authors' Contributions}

Jie Yang designed and performed the study, collected and analyzed the data, and wrote and revised the manuscript. Yan Kang designed and performed the study and wrote and revised the manuscript. Yongfang Zhou designed and 
performed the study and wrote the manuscript. Binbin $\mathrm{Xu}$ performed the study, analyzed the data, and revised the manuscript. Peng Wang, Yinxia Lv, and Zhen Wang collected and analyzed the data and assisted in writing the manuscript. All authors read and approved the final manuscript.

\section{Acknowledgments}

The authors express their gratitude to all those who helped them during this study. They are particularly grateful to their respiratory therapist team for their timely help. They also thank the nurses and their colleagues in the Department of Critical Care Medicine, West China Hospital of Sichuan University.

\section{References}

[1] E. W. Ely, S. K. Inouye, G. R. Bernard et al., "Delirium in mechanically ventilated patients: validity and reliability of the confusion assessment method for the intensive care unit (CAMICU)," The Journal of the American Medical Association, vol. 286, no. 21, pp. 2703-2710, 2001.

[2] J. I. Salluh, M. Soares, J. M. Teles et al., "Delirium epidemiology in critical care (DECCA): an international study," Critical Care, vol. 14, no. 6, article R210, 2010.

[3] J. Potter and J. George, "The prevention, diagnosis and management of delirium in older people: Concise guidelines," Clinical Medicine (London, England), vol. 6, no. 3, pp. 303-308, 2006.

[4] D. Kukreja, U. Günther, and J. Popp, "Delirium in the elderly: Current problems with increasing geriatric age," Indian Journal of Medical Research, vol. 142, no. 6, pp. 655-662, 2015.

[5] S. B. Bryczkowski, M. C. Lopreiato, P. P. Yonclas, J. J. Sacca, and A. C. Mosenthal, "Risk factors for delirium in older trauma patients admitted to the surgical intensive care unit," Journal of Trauma and Acute Care Surgery, vol. 77, no. 6, pp. 944-951, 2014.

[6] S. Arumugam, A. El-Menyar, A. Al-Hassani et al., "Delirium in the intensive care unit," Journal of Emergencies, Trauma, and Shock, vol. 10, no. 1, pp. 37-46, 2017.

[7] E. Ely, S. Gautam, R. Margolin et al., "The impact of delirium in the intensive care unit on hospital length of stay," Intensive Care Medicine, vol. 27, no. 12, pp. 1892-1900, 2001.

[8] E. W. Ely, A. Shintani, B. Truman et al., "Delirium as a predictor of mortality in mechanically ventilated patients in the intensive care unit," The Journal of the American Medical Association, vol. 291, no. 14, pp. 1753-1762, 2004.

[9] E. B. Milbrandt, S. Deppen, P. L. Harrison et al., "Costs associated with delirium in mechanically ventilated patients," Critical Care Medicine, vol. 32, no. 4, pp. 955-962, 2004.

[10] C. N. Sessler and W. Wilhelm, "Analgesia and sedation in the intensive care unit: An overview of the issues," Critical Care, vol. 12, no. 3, article no. S1, 2008.

[11] R. I. Hall, D. Sandham, P. Cardinal et al., "Propofol vs midazolam for ICU sedation: A Canadian multicenter randomized trial," CHEST, vol. 119, no. 4, pp. 1151-1159, 2001.

[12] H. Wunsch, J. M. Kahn, A. A. Kramer, and G. D. Rubenfeld, "Use of intravenous infusion sedation among mechanically ventilated patients in the United States," Critical Care Medicine, vol. 37, no. 12, pp. 3031-3039, 2009.

[13] J. Barr, G. L. Fraser, K. Puntillo et al., "Clinical practice guidelines for the management of pain, agitation, and delirium in adult patients in the intensive care unit," Critical Care Medicine, vol. 41, no. 1, pp. 263-306, 2013.

[14] W. B. Cammarano, J.-F. Pittet, S. Weitz, R. M. Schlobohm, and J. D. Marks, "Acute withdrawal syndrome related to the administration of analgesic and sedative medications in adult intensive care unit patients," Critical Care Medicine, vol. 26, no. 4, pp. 676-684, 1998.

[15] A. Shafer, "Complications of sedation with midazolam in the intensive care unit and a comparison with other sedative regimens," Critical Care Medicine, vol. 26, no. 5, pp. 947-956, 1998.

[16] Y. Zhou, X. Jin, Y. Kang, G. Liang, T. Liu, and N. Deng, "Midazolam and propofol used alone or sequentially for long-term sedation in critically ill, mechanically ventilated patients: A prospective, randomized study," Critical Care, vol. 18, no. 3, article no. R122, 2014.

[17] C. N. Sessler, M. S. Gosnell, M. J. Grap et al., "The Richmond Agitation-Sedation Scale: validity and reliability in adult intensive care unit patients," American Journal of Respiratory and Critical Care Medicine, vol. 166, no. 10, pp. 1338-1344, 2002.

[18] J.-L. Vincent, Y. Shehabi, T. S. Walsh et al., "Comfort and patient-centred care without excessive sedation: the eCASH concept," Intensive Care Medicine, vol. 42, no. 6, pp. 962-971, 2016.

[19] T. D. Girard, J. P. Kress, B. D. Fuchs et al., "Efficacy and safety of a paired sedation and ventilator weaning guideline for mechanically ventilated patients in intensive care (Awakening and Breathing Controlled trial): a randomised controlled trial," The Lancet, vol. 371, no. 9607, pp. 126-134, 2008.

[20] J.-M. Boles, J. Bion, A. Connors et al., "Weaning from mechanical ventilation," European Respiratory Journal, vol. 29, no. 5, pp. 1033-1056, 2007.

[21] B. Van Rompaey, M. M. Elseviers, M. J. Schuurmans, L. M. Shortridge-Baggett, S. Truijen, and L. Bossaert, "Risk factors for delirium in intensive care patients: a prospective cohort study," Critical Care, vol. 13, no. 3, article no. R77, 2009.

[22] S. Mehta, D. Cook, J. W. Devlin et al., "Prevalence, risk factors, and outcomes of delirium in mechanically ventilated adults," Critical Care Medicine, vol. 43, no. 3, pp. 557-566, 2015.

[23] E. W. Steyerberg, M. J. C. Eijkemans, F. E. Harrell Jr., and J. D. F. Habbema, "Prognostic modelling with logistic regression analysis: a comparison of selection and estimation methods in small data sets," Statistics in Medicine, vol. 19, no. 8, pp. 10591079, 2000.

[24] J. A. McPherson, C. E. Wagner, L. M. Boehm et al., "Delirium in the cardiovascular ICU: exploring modifiable risk factors," Critical Care Medicine, vol. 41, no. 2, pp. 405-413, 2013.

[25] C. B. Weaver, S. L. Kane-Gill, S. R. Gunn, L. Kirisci, and P. L. Smithburger, "A retrospective analysis of the effectiveness of antipsychotics in the treatment of ICU delirium," Journal of Critical Care, vol. 41, pp. 234-239, 2017.

[26] S.-H. Wang, J.-Y. Wang, P.-Y. Lin et al., "Predisposing risk factors for delirium in living donor liver transplantation patients in intensive care units," PLoS ONE, vol. 9, no. 5, Article ID e96676, 2014.

[27] S. B. Patel, J. T. Poston, A. Pohlman, J. B. Hall, and J. P. Kress, "Rapidly reversible, sedation-related delirium versus persistent delirium in the intensive care unit," American Journal of Respiratory and Critical Care Medicine, vol. 189, no. 6, pp. 658665, 2014. 
[28] E. Arend and M. Christensen, "Delirium in the intensive care unit: a review," Nursing in Critical Care, vol. 14, no. 3, pp. 145154, 2009.

[29] S. A. Park, Y. Tomimaru, A. Shibata, S. Miyagawa, K. Noguchi, and K. Dono, "Incidence and risk factors for postoperative delirium in patients after hepatectomy," World Journal of Surgery, vol. 41, no. 11, pp. 2847-2853, 2017.

[30] C. Shi, C. Yang, R. Gao, and W. Yuan, "Risk Factors for Delirium after Spinal Surgery: A Meta-Analysis," World Neurosurgery, vol. 84, no. 5, pp. 1466-1472, 2015.

[31] S. Ouimet, B. P. Kavanagh, S. B. Gottfried, and Y. Skrobik, "Incidence, risk factors and consequences of ICU delirium," Intensive Care Medicine, vol. 33, no. 1, pp. 66-73, 2007.

[32] P. Pandharipande, A. Shintani, J. Peterson et al., "Lorazepam is an independent risk factor for transitioning to delirium in intensive care unit patients," Anesthesiology, vol. 104, no. 1, pp. 21-26, 2006.

[33] J.-L. Vincent, R. Moreno, J. Takala et al., "The SOFA (Sepsisrelated Organ Failure Assessment) score to describe organ dysfunction/failure," Intensive Care Medicine, vol. 22, no. 7, pp. 707-710, 1996.

[34] W. A. Knaus, E. A. Draper, D. P. Wagner, and J. E. Zimmerman, "APACHE II: a severity of disease classification system," Critical Care Medicine, vol. 13, no. 10, pp. 818-829, 1985.

[35] G. L. Fraser, J. W. Devlin, C. P. Worby et al., "Benzodiazepine versus nonbenzodiazepine-based sedation for mechanically ventilated, critically ill adults: A systematic review and metaanalysis of randomized trials," Critical Care Medicine, vol. 41, no. 9, pp. S30-S38, 2013.

[36] G. L. Weinhouse and P. L. Watson, "Sedation and Sleep Disturbances in the ICU," Critical Care Clinics, vol. 25, no. 3, pp. 539-549, 2009.

[37] D. M. Wade, D. C. Howell, J. A. Weinman et al., "Investigating risk factors for psychological morbidity three months after intensive care: a prospective cohort study," Critical Care, vol. 16, no. 5, article no. R192, 2012.

[38] V. Agarwal, P. J. O’Neill, B. A. Cotton et al., "Prevalence and risk factors for development of delirium in burn intensive care unit patients," Journal of Burn Care \& Research, vol. 31, no. 5, pp. 706$715,2010$.

[39] P. Pandharipande, B. A. Cotton, A. Shintani et al., "Prevalence and risk factors for development of delirium in surgical and trauma intensive care unit patients," The Journal of Trauma and Acute Care Surgery, vol. 65, no. 1, pp. 34-41, 2008.

[40] Z.-Q. Xia, S.-Q. Chen, X. Yao, C.-B. Xie, S.-H. Wen, and K.-X. Liu, "Clinical benefits of dexmedetomidine versus propofol in adult intensive care unit patients: A meta-analysis of randomized clinical trials," Journal of Surgical Research, vol. 185, no. 2, pp. 833-843, 2013.

[41] G. Djaiani, N. Silverton, L. Fedorko et al., "Dexmedetomidine versus Propofol Sedation Reduces Delirium after Cardiac Surgery: A Randomized Controlled Trial," Anesthesiology, vol. 124, no. 2, pp. 362-368, 2016.

[42] X. Su, Z.-T. Meng, X.-H. Wu et al., "Dexmedetomidine for prevention of delirium in elderly patients after non-cardiac surgery: a randomised, double-blind, placebo-controlled trial," The Lancet, vol. 388, no. 10054, pp. 1893-1902, 2016.

[43] P. M. Szumita, S. A. Baroletti, K. E. Anger, and M. E. Wechsler, "Sedation and analgesia in the intensive care unit: Evaluating the role of dexmedetomidine," American Journal of HealthSystem Pharmacy, vol. 64, no. 1, pp. 37-44, 2007.
[44] S. M. Jakob, E. Ruokonen, R. M. Grounds et al., "Dexmedetomidine vs midazolamor propofol for sedation during prolonged mechanical ventilation: two randomized controlled trials," The Journal of the American Medical Association, vol. 307, no. 11, pp. 1151-1160, 2012. 


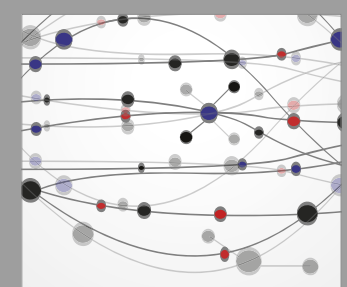

The Scientific World Journal
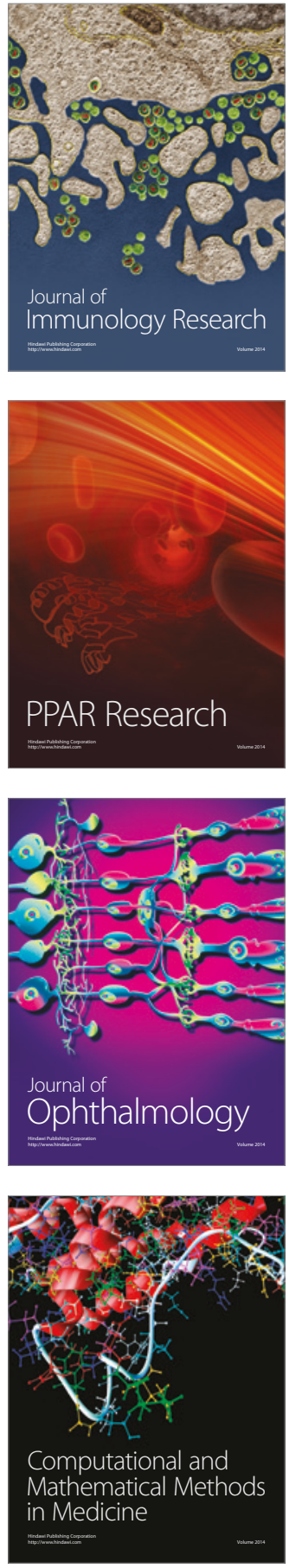

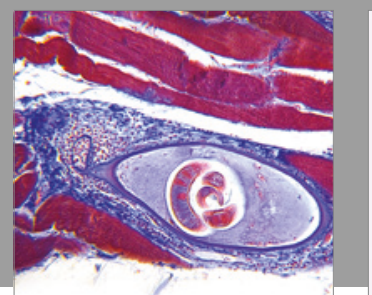

Gastroenterology Research and Practice
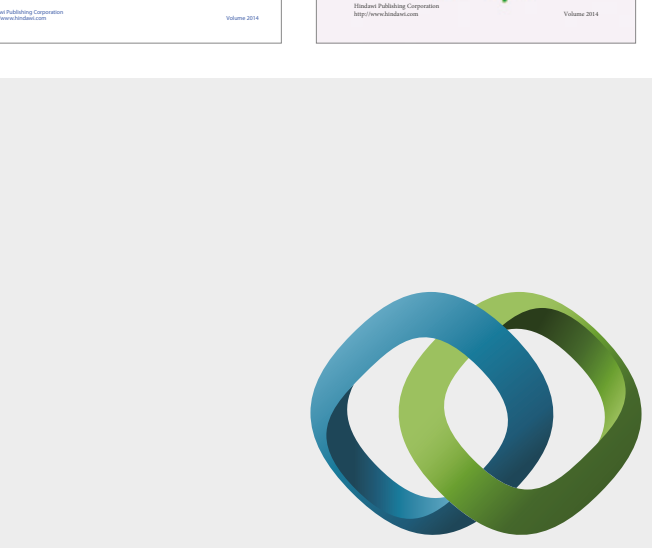

\section{Hindawi}

Submit your manuscripts at

https://www.hindawi.com
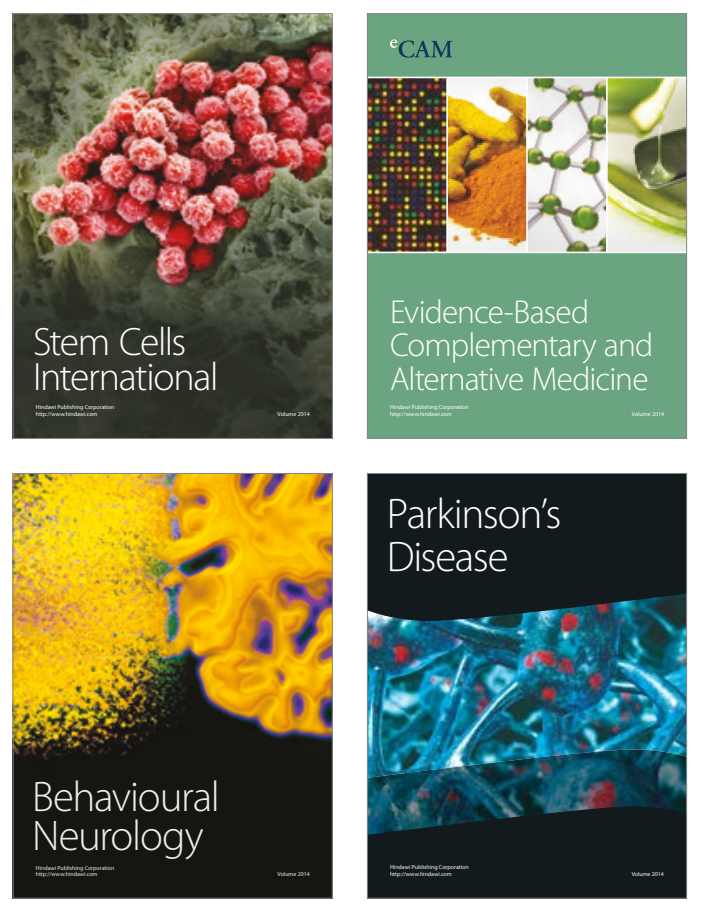
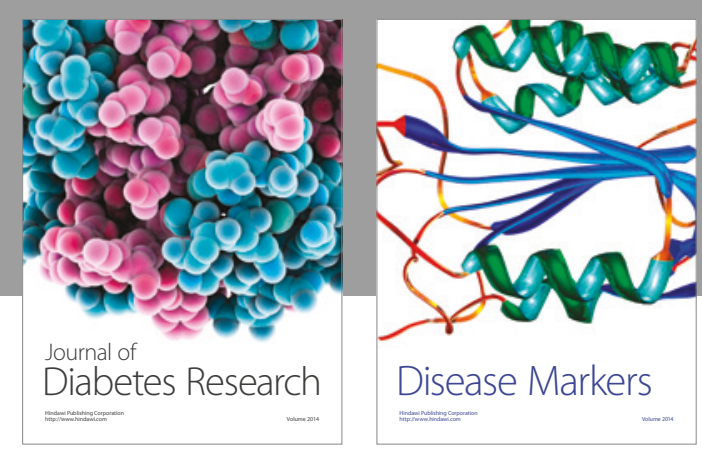

Disease Markers
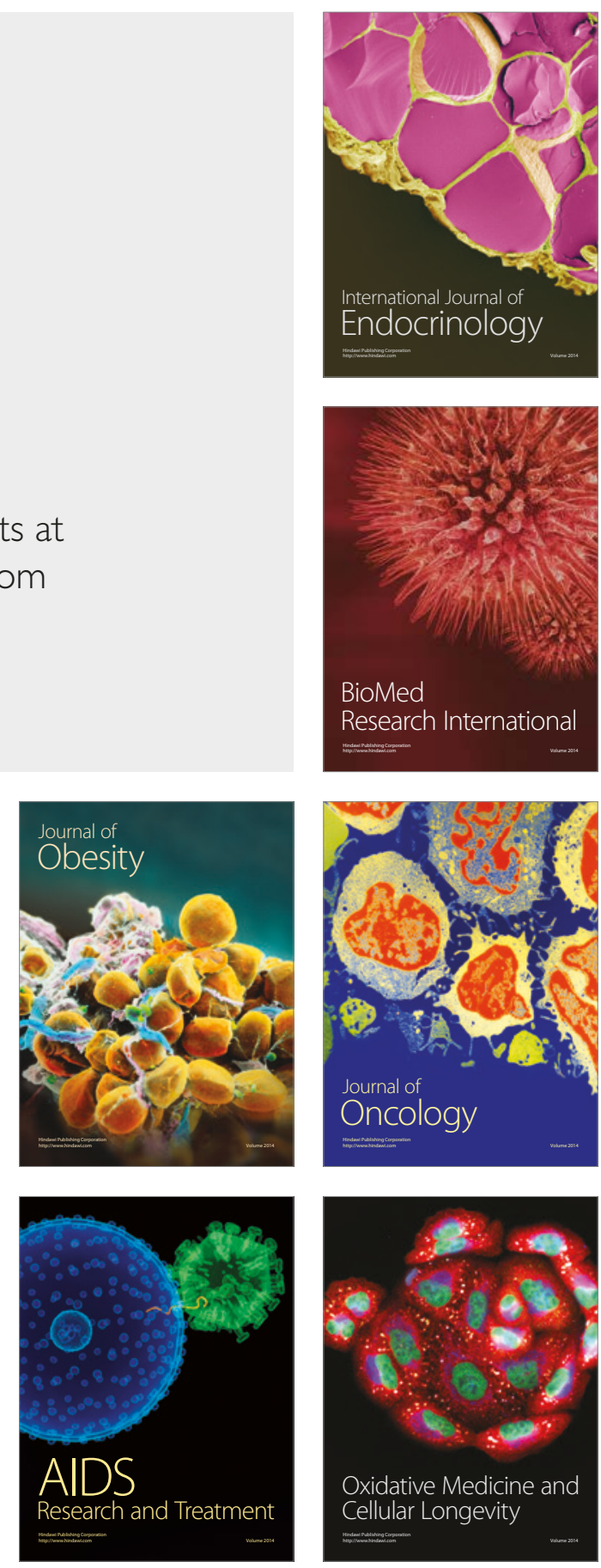